\title{
EPIDEMIOLOGIA DA HIPERTENSÃO ARTERIAL SISTÊMICA
}

\author{
EPIDEMIOLOGY OF HYPERTENSION
}

Nelson Spritzer

Mestre em Cardio (Universidade Federal do Rio Grande do Sul); Doutor em Nefro (Escola Paulista de Medicina);Diretor da Cardiopress - RS. CoRRESPONDÊNCIA: Nelson Spritzer - Rua Bernardes Vieira, 209/402 - Porto Alegre - RS

SPRITZER N. Epidemiologia da hipertensão arterial sistêmica. Medicina, Ribeirão Preto, 29: 199-213, abr./set. 1996.

RESUMO: Neste artigo, são discutidos os dados de prevalência de hipertensão arterial sistêmica, especialmente aqueles oriundos de estudos baseados em comunidades. São avaliadas as diferenças de prevalência tanto da própria hipertensão como de suas repercussões clínicas mais freqüentes, incluindo mortalidade. Os dados são discutidos com especial atenção às distinções quanto às raças, sexos, faixas de idade e condição sócioeconômica, medida pela escolaridade e as implicações sobre a prevenção primária.

UNITERMOS: Hipertensão. Doenças Cardiovasculares.

\section{INTRODUÇÃO}

A variação populacional da ocorrência e seqüelas da hipertensão arterial sistêmica (HAS), relacionadas às características de raça, sexo, idade é tão comum e mensurável nas sociedades industrializadas que os efeitos destes atributos na hipertensão são, geralmente, considerados como fixos e resultantes de processos biológicos, os quais não podem ser modificados pela exposição ambiental usual ou por experiências de vida. A evidência epidemiológica, entretanto, indica que a associação entre hipertensão e idade, sexo e raça não é casual dentro de uma população e que a designação raça é freqüentemente um indicador secundário de nível socioeconômico. Assim, enquanto estas características refletem diferenças quanto ao comportamento, estilo de vida e exposição ambiental, as quais por si só estão relacionadas à HAS, haverá diferenças no risco de desenvolvimento da HAS, na severidade do processo e no prognóstico. Por conseguinte, a associação de HAS com idade, raça e sexo refletirá a inter-relação entre os chamados fatores ambientais e os genéticos. Por exemplo, a associação de elevação da pressão arterial com o sobrepeso está bem documentada. $\mathrm{O}$ aumento de peso corporal com o aumento da idade, observados nas sociedades desenvolvidas são responsáveis por uma proporção do crescimento na prevalência de HAS em idosos. Similarmente, estudos sobre migrações populacionais, nas quais a transição de um estilo de vida mais ativo para um mais sedentário, com concomitante aumento da incidência da obesidade com a idade mostram um aumento concomitante na freqüência de HAS. Em adição à elevação da pressão populacional com a idade, existem também diferenças nas mudanças e nos níveis de mudança, entre populações e dentro de uma mesma população. As populações diferem e podem ser classificadas por estes atributos, considerando as mudanças e os níveis de mudanças conforme a idade.

A ênfase colocada sobre o "status socioeconômico" deriva de sua utilidade nos estudos epidemiológicos como um índice das diferenças entre indivíduos expostos às experiências ambientais e de vida. Sua capacidade em sumariar um conjunto de 
diferentes fatores, cada qual potencialmente relevante à saúde e à doença, faz seu estudo útil em análises descritivas e para seguimentos prospectivos populacionais. Sua natureza composta faz deste conjunto de informações menos útil para investigações epidemiologicamente orientadas. Estes atributos de alguns indivíduos, em relação a outros, dentro de suas sociedades coloca-os por prestígio e sumariza suas possessões e comando de recursos, dentro de suas comunidades com potenciais consequiências para risco de doenças e seu tratamento.

O material que se segue é baseado primariamente em estudos epidemiológicos, conduzidos em países desenvolvidos, notadamente, nos Estados Unidos da América onde a qualidade e quantidade de dados populacionais é exuberante e confiável. Tais dados sumarizam as evidências relacionando o "status socioeconômico" e a HAS, bem como suas seqüelas entre os indivíduos considerados; entretanto, comparações com dados do nosso País poderão ser incluídas quando apropriadas e/ou confiáveis. A organização do material deste capítulo segue o seguinte formato: Uma introdução que coloca as informações epidemiológicas mais gerais, segue-se os dados de estudos "observacionais", descrevendo a prevalência da HAS e sua relação com as características de interesse (idade, sexo, raça, peso, etc...), o prognóstico da HAS refletido pelo risco de mortalidade, influenciado por estes fatores e pelo cuidado médico dispensado (epidemiologia clínica), a modificação da história natural e do curso clínico por intervenções farmacológicas e não farmacológicas, entre os indivíduos em diferentes estratos socioeconômicos e finalmente, as evidências destas relações como agregadoras, dentro de uma população ou comunidade.

A HAS, definida pelos propósitos clínicos, requer que os indivíduos se encaixem em critérios clínicos e diagnósticos bem específicos. Atualmente, estes critérios seguem baseados em diversas medições de pressão arterial, as quais levam, pela aplicação de tais critérios, a uma especificação categórica, qualitativa e absoluta. Esta forma de definição é, sem dúvida, arbitrária, do ponto de vista da epidemiologia. Em adição à considerável variabilidade intra-individual da pressão arterial, o que significa que a maioria dos indivíduos, quando examinada em diferentes ocasiões, poderá preencher os critérios de normalidade e/ou HAS em diferentes períodos. Existe, também, a arbitrária dicotomia de uma distribuição contínua da pressão arterial em uma população. Isso é feito a des- peito da contínua distribuição da pressão em um mesmo indivíduo, dentro de uma população sem evidências de multimodalidade. Existe um problema adicional, resultante da categorização dos indivíduos, resultado da natureza contínua e variável do fenômeno, pressão arterial, relacionado ao risco cardiovascular. A função risco é contínua, elevando-se em proporção direta à medida em que notamos a elevação da pressão arterial, sem uma clara evidência da existência de um limiar ou ponto de corte, onde se pode dizer com certeza que dali em diante há risco e abaixo do qual o risco inexiste. Essa variável contínua existe, por exemplo, para eventos tais como os acidentes vasculares encefálicos, insuficiência cardíaca congestiva, coronariopatia e mortalidade por todas as causas. A função do risco cresce mais do que seria de se esperar, por uma forma linear comparada à crescente pressão arterial e a inclinação de sua curva ascendente que se acelera com a elevação da pressão. A conseqüência é que o risco de sequielas e danos é muito maior nos níveis mais elevados de pressão arterial e é, relativamente, baixo nos estratos mais próximos da média populacional.

Em contraste com às implicações individuais, o impacto populacional de elevações modestas da pressão arterial é maior em níveis de distribuição da pressão arterial bem próximos da média populacional. O maior impacto populacional "de menos severas elevações de pressão" reflete o maior número de indivíduos incluídos no estrato de risco, a despeito de seus baixos perfis de risco individuais, em oposição com a menor quantidade de indivíduos, com maior risco individual e elevações marcadas da pressão arterial. Essa diferença, no impacto populacional, conduz a diferentes recomendações para indivíduos em maior risco, ao contrário de outros com mais modestas elevações de pressão, o que faz diferença nos programas para os indivíduos de alto risco versus programas populacionais, quando se estuda estratégias de controle da HAS. As últimas requerem estudos visando uma mudança da curva de distribuição populacional para valores de pressão mais reduzidos. Estas perspectivas populacionais, enquanto centrais para a compreensão epidemiológica do controle da HAS, na população, não serão exploradas em profundidade, neste capítulo. O foco será na HAS, como definida pela clínica. O efeito modificador do "status socioeconômico" na associação da idade, sexo e, particularmente, raça na prevalência e conseqüências da HAS terá especial atenção. 
A associação entre HAS e idade para adultos, entre 18 e 74 anos, está sumarizada neste capítulo. Os contrastes com relação à raça serão aqueles entre brancos e não brancos. A associação da HAS e indivíduos negros e sua maior severidade merecerá atenção específica. A disponibilidade de dados raciais é expressiva quanto aos brancos e negros e, por esta razão, serão considerados com maior interesse.

Antes de revisar a associação entre os fatores socioeconômicos e a hipertensão, é construtivo considerar que uma relação inversa de uma série de outras doenças têm sido bem documentadas em vários contextos, aliados a tais fatores dentro e entre nações. A consistência e a universalidade da relação inversa entre saúde e "status socioeconômico" com relação à pessoa, lugar e tempo, já foi adequadamente documentada. Isso foi relatado para indivíduos de diferentes idades, em diferentes períodos de tempo e em diferentes países. O processo que está relacionado a estas associações são múltiplos e diversos quanto a sua natureza, estendendo-se do comportamento individual até os domínios do ambiente psicoquímico.A relação entre o status social e a saúde apesar de usualmente inversa e presente em sociedades de todos os níveis de desenvolvimento social e econômico, ocasionalmente está ausente ou é de natureza discreta. Por exemplo, a associação entre o status socioeconômico e a mortalidade por doença coronariana, era aparentemente direta e mais alta nos estratos mais elevados da população, até meados deste século no Reino Unido. Nos últimos dez anos, inverteu-se tal tendência e, agora, é exatamente no estrato mais alto que existem os menores índices de mortalidade coronariana comparada aos dos estratos inferiores da sociedade. Um evento similar ocorreu num estudo com populações rurais no estado da Georgia (USA).

As potenciais contribuições dos fatores sociais e ambientais têm sido investigadas por vários pesquisadores, enquanto outros têm enfatizado a importância da resistência individual, recursos e respostas fisiológicas baseadas em respostas condicionadas ou aprendidas. Entre as explicações invocadas para explicar os processos responsáveis para as associações observadas entre o status socioeconômico e HAS estão aquelas do estresse psicossocial, diferenças alimentares e de atividades físicas, massa corporal e diferenças na aceitação de, ou da utilização, dos cuidados de saúde. Não é o propósito desta discussão rever as contribuições de cada um destes ou outros possíveis mecanismos exploratórios. Ao contrário, a intenção é de apresentar uma revisão das evidências empíricas da associação entre o status socioeconômico e a HAS em estágios que vão da sua prevalência até a epidemiologia clínica, as seqüelas deletérias individuais e os índices de mortalidade nos EUA. A modificação da associação das seqüelas hipertensivas relacionadas com o status socioeconômico, é de particular interesse para a terapia anti-hipertensiva.

Conquistas educacionais são usadas como índices ou marcadores secundários de status socioeconômico. É o mais acessível indicador epidemiológico (com validade demonstrada em países do primeiro mundo) que possibilita perceber o status socioeconômico nos seus vários aspectos, de forma global (p. ex. prestígio, poder, acesso a recursos materiais, controle sobre mudanças de vida num ambiente material e social).

\section{HIPERTENSÃO EM NEGROS}

A desvantagem dos negros tem sido bem demonstrada para a maioria das condições de saúde estudadas. Essa iniqüidade tem sido particularmente notada na HAS e suas seqüelas. Tem havido escassos estudos verificando a condição socioeconômica em populações negras, quanto à prevalência da HAS nestes grupos, apesar de que os poucos estudos, com este teor, apontam para uma maior agregação dos negros do que dos brancos, em relação à HAS, independente do seu status socioeconômico. Isso parece ser verdadeiro para a doença cardiovascular, em geral, e para a HAS, em particular, uma conseqüência da estratégia usada na pesquisa diferente da usada em grupos de negros e de brancos, entre as quais são incluídos vários estudos concernentes ao status socioeconômico. A tendência geral é de caracterizar populações negras como homogêneas. Isso resulta de pelo menos duas considerações: primeiro, a marcada diferença dos negros quanto ao status social, ocupacional e econômico, em relação aos brancos. Até recentemente, quase $95 \%$ dos negros norte-americanos ficavam classificados, quanto ao "status socioeconômico" bem abaixo da média atingida pelos brancos. Segundo, a história social dos negros, notadamente nos EUA, resultou numa crença compartilhada pelos pesquisadores de que os negros são indivíduos mais suscetíveis às doenças por ordem de fatores genéticos. Um dos principais propósitos desta apresentação será o de, empiricamente, ilustrar, com a experiência de um grande 
estudo, o Hypertension Detection and Follow-Up Program (HDFP), de que o conhecimento do "status socioeconômico", mais do que a raça, nos dá informações com valor preditivo sobre a prevalência, severidade e sequielas da HAS, tanto em negros como em brancos e que o prognóstico pode ser modificável (e possivelmente, pode ser eliminado) por terapêutica anti-hipertensiva eficaz e efetiva. $\mathrm{O}$ atributo cor da pele, por si só, impõe uma dimensão de status e esta situação é fundamental na compreensão da epidemiologia da HAS em nosso meio.

$\mathrm{O}$ achado de elevada prevalência de HAS em negros, com altos níveis de pressão arterial, comparados aos brancos, tem sido reportado em numerosos estudos nos EUA e Reino Unido. Em contraste, resultados conflitantes têm sido observados em estudos com africanos, residentes em áreas rurais. Os problemas metodológicos em definir a demografia destas populações, fazendo amostras expressivas e fazendo determinações de pressão arterial, com adequada estandardização, são formidáveis e têm impedido que se extraia conclusões firmes quando se compara os dados destes estudos. A questão da natureza da distribuição dos níveis de pressão arterial, entre os negros residentes em seus lugares de nascimento originais, antes da experiência da emigração forçada pela escravidão, permanece uma questão em aberto. Entretanto, evidências mais recentes como as discutidas anteriormente, nos dão a clara idéia da marcada variação nos níveis de pressão arterial e na prevalência da HAS entre negros em relação às experiências e exposições ao ambiente socioeconômico.

\section{RAÇA, GENÉTICA, "STATUS SOCIOECONÔMI- CO” E HIPERTENSÃO ARTERIAL SISTÊMICA}

Existem evidências de agregação intrafamiliar dos níveis de pressão arterial e de prevalência de HAS numa maneira consistente, com determinantes genéticos de HAS dentro das populações; p. ex. o grau de similaridade intrafamiliar é maior para parentes biológicos do que para os não biológicos numa mesma família. Essas observações não explicam as diferenças entre populações. Há uma base genética para algumas características fenotípicas (ex. cor de pele) usada para classificar indivíduos por raça. Uma confusão comum é assumir tais traços como HAS os quais também variam entre os grupos classificados racialmente como distintos, e que suportam a hipótese da explicação genética para tais diferenças. Um estudo, p. ex., acessou a associação entre a quantidade de pigmentação da pele (determinada pela medida da cor de pele) e os níveis de pressão arterial em negros. Neste estudo, houve uma correlação positiva entre a cor da pele e os níveis de pressão arterial, tal que um discreto aumento na pigmentação da pele seguir-se-ia de uma notável elevação da pressão arterial. Assumir que isso é decorrente de determinação genética ou biológica, entretanto, seria ignorar que houve uma associação entre a cor da pele e mudanças de vida bem determinada, no sul dos EUA nos anos 60; as exposições às experiências de vida das pessoas de classes mais baixas, negros, foram bem distintas das dos de classes mais elevadas, usualmente gente de cor mais clara. Um estudo subseqüente, dentro da mesma população, mostrou uma forte associação entre cor de pele e status socioeconômico, e quando se controlava a variável social não restava mais nenhuma associação entre a cor da pele e os níveis de pressão arterial elevados. Inversamente, mantendo a cor da pele como constante, houve uma correlação inversa entre a incidência de HAS e o status socioeconômico. Achados como estes não refutam a importância dos fatores genéticos na etiopatogenese da HAS para cada indivíduo. Apesar de que mecanismos mais precisos estão para ser identificados, seria uma conjetura verossímil dizer que existem mecanismos moleculares, celulares e fisiológicos responsáveis pela elevação da pressão arterial, os quais poderão ser melhor esclarecidos numa base molecular, tanto para a hipertensão, como para tantas outras condições biológicas. Não se conclui disso que o genótipo necessário para HAS será encontrado como sendo, diferentemente, distribuído entre populações caracterizadas com base nos traços usados para classificações sociais e raciais dos indivíduos. E na possibilidade remota de que assim seja, é também verdadeiro que a expressão da HAS requererá a exposição a fatores ambientais bem relevantes. É bem possível que tal exposição seja bem diferente entre brancos e negros, numa mesma população.

Estimativas da associação entre os índices educacionais (como indicadores de status socioeconômico) com a HAS, suas conseqüências e a prevenção, obtidas como resultado do adequado tratamento, estão sumariadas a seguir, com dados baseados nas pesquisas, tais como National Health and Nutrition Survey (NHANES I e II - 1976-1980), o HDFP (1974-1979) e os índices de mortalidade norte-americanos. 


\section{ESTIMATIVAS DE PREVALÊNCIA}

\subsection{Prevalência nos EUA}

Estimativas da distribuição dos níveis de pressão arterial para os EUA têm sido derivadas, periodicamente, de estudos de coortes conduzidos pelo National Center for Health Statistics. As informações mais recentes, reportadas por este órgão foram obtidas durante o período de 1976 a 1980 através do National Health and Nutrition Examination Survey II (NHANES II), baseadas numa amostra representativa nacional de pessoas não institucionalizadas dos Estados Unidos. A pressão arterial foi medida, indiretamente, por esfigmomanometro de mercúrio com estandardização das circunstâncias das medidas, número de medições e treinamento dos observadores - fatores muito importantes para que tivessem sido obtidos dados confiáveis. Cabe comentar que os poucos estudos epidemiológicos conduzidos no Brasil, na área da hipertensão, careceram dos cuidados básicos e padrões de qualidade mínimos para se confiar nos resultados obtidos, por isso, estamos apresentando os dados norte-americanos. Os dados de medidas de pressão arterial foram as médias de três medidas. As dificuldades de medições múltiplas em diferentes ocasiões, em estudos populacionais, são evidentes.

Níveis de pressão arterial sistólica e diastólica variam com a idade. As médias de pressão, relatadas no NHANES II, para adultos do sexo feminino, com idade entre 18-74 anos estão mostradas na Figura 1, em intervalos de 10 anos, tanto para brancas como para negras. O intervalo de confiança de $95 \%$ ao redor da média estão marcados para cada medida, mostrando a faixa onde se inclui os valores verdadeiros para todo o País, a partir desta amostra. Não há entrecruzamento para estes intervalos de confiança para a maioria dos valores, observados nas negras em contraste com os das brancas, confirmando o achado, freqüentemente, reportado de que existe maior prevalência de hipertensão arterial de níveis de pressão elevados nas negras do que nas brancas.

A associação dos níveis de pressão arterial, com a idade, difere entre as medidas diastólicas e as sistólicas. As médias sistólicas crescem com a idade de forma regular e contínua. As médias diastólicas crescem até cerca dos 50 anos, seguindo-se de um platô e um decréscimo com as faixas etárias mais elevadas. Isso é verdadeiro tanto em brancas como em negras. Essas observações são baseadas em sobreviventes num determinado período de tempo. A possível razão do declínio das diastólicas, após a meia idade, inclui explicações tais como seleção natural dos sobreviventes mais saudáveis. A discordância de trajetórias das sistólicas e diastólicas, com o avançar da idade, produz maior prevalência de hipertensão sistólica isolada nos mais idosos.

Quanto aos homens, a pressão arterial se comporta de maneira similar ao que aconteceu com as mulheres. Há uma ascensão das sistólicas com a

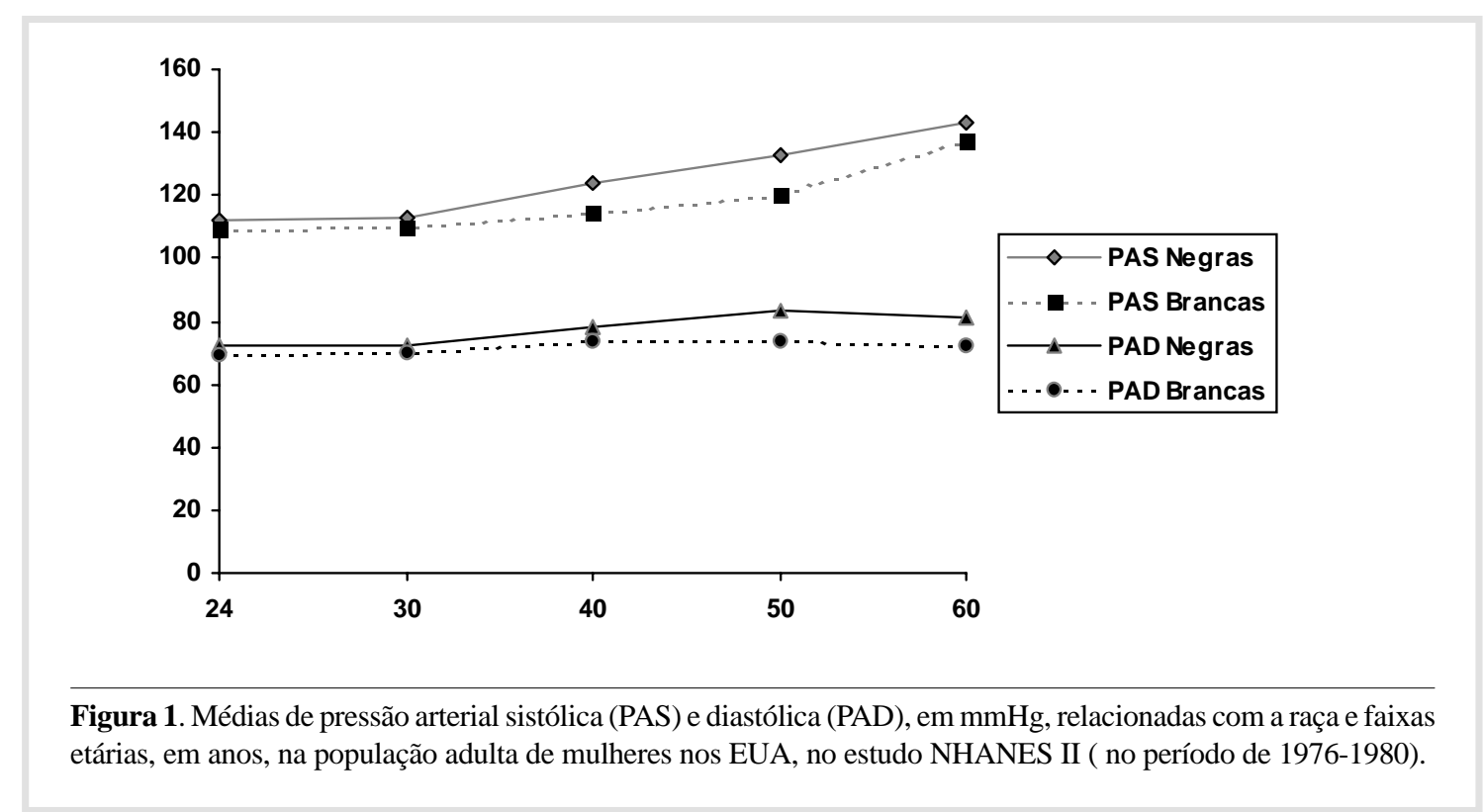


idade, o que é contínuo e regular, enquanto que as diastólicas, ao redor dos 50 anos tendem ao platô ou decréscimo. Apesar de que os valores médios são mais elevados nos pretos do que nos brancos, após os 30 anos, isso não é significativo do ponto de vista estatístico (Figura 2).

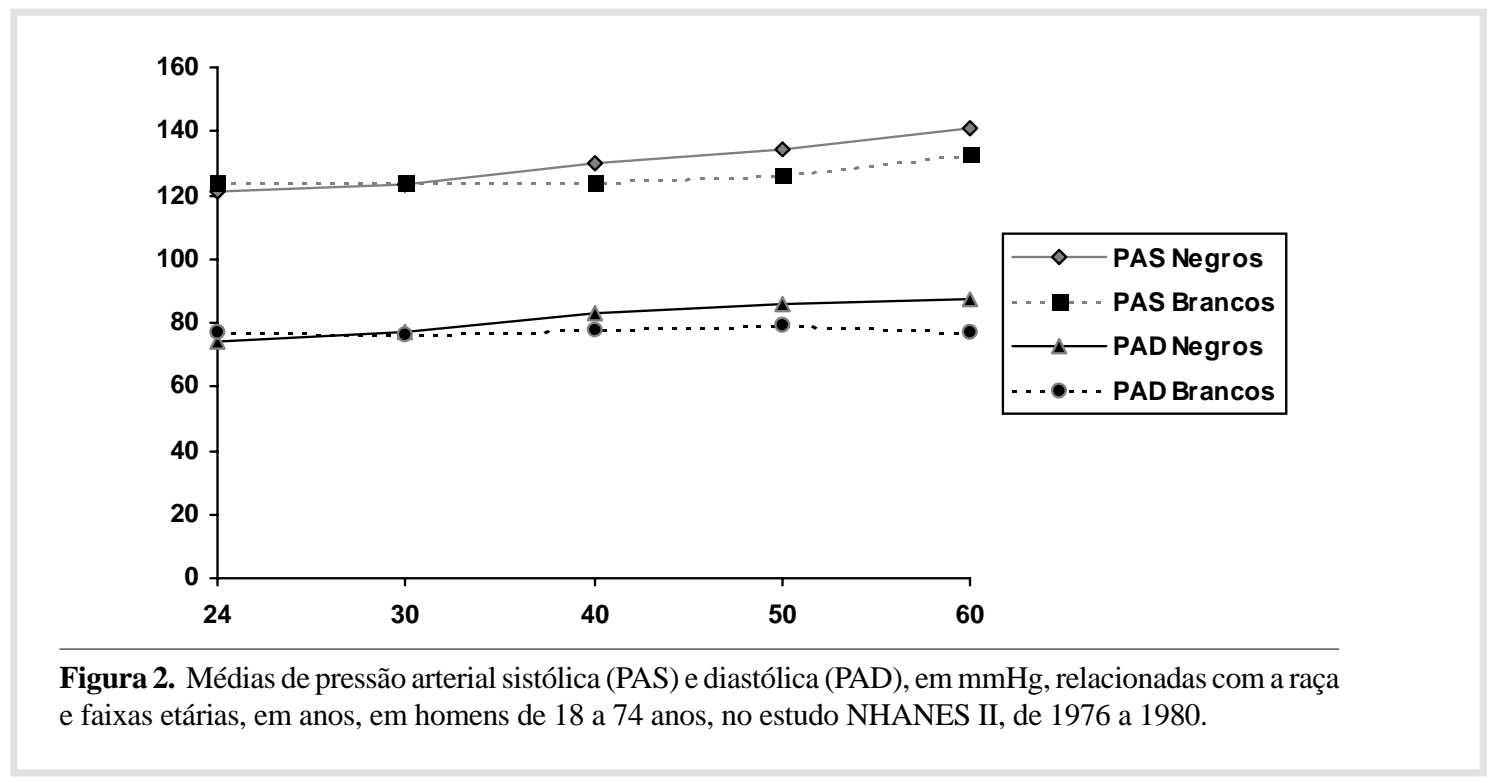

\subsubsection{O Hypertension Detection and Follow-up Program (HDFP)}

O HDFP foi um estudo baseado em comunidades, com 14 centros. Foi feita uma tentativa de se alcançar pessoas portadoras de hipertensão dentro das comunidades estudadas para verificar-se a eficácia de um programa de tratamento farmacológico chamado, stepped-care (tratamento escalonado) no controle da pressão arterial. O ponto terminal do estudo era a redução da mortalidade por todas as causas e redução nos danos em órgãos-alvos selecionados. O HDFP não foi desenhado para ser uma amostra nacional representativa de toda a população norte-americana; entretanto, as 14 comunidades escolhidas foram, geograficamente, bem dispersas, heterogêneas quanto a sua formação demográfica. Além disso, houve uma intencional inclusão superestimada de elementos negros em todas as amostras. Mais de 150.000 pessoas tiveram suas pressões arteriais medidas em casa para a identificação de possíveis candidatos à randomização para o estudo. Indivíduos com diastólica, sustentada acima de $95 \mathrm{mmHg}$, eram avaliadas numa segunda ocasião e os que permaneciam igual ou acima de $90 \mathrm{mmHg}$ eram randomizados para clínicas onde recebiam o tratamento escalonado ou o tratamento regular. O tratamento escalonado foi desenhado como uma forma sistemática de tratamento farmacológico para alcançar-se redução eficaz dos níveis de pressão arterial, atingindo níveis preestabelecidos como metas terapêuticas. A lógica era aumentar as doses de determinada droga inicial, em geral, diuréticos, caso não alcançasse o resultado desejado, uma segunda droga era incluída, em geral um betabloqueador, e suas doses elevadas até a resposta desejada ou uma terceira associação. $\mathrm{O}$ seguimento foi feito por 5 anos. Dados como adesão ao tratamento, níveis de pressão arterial, estado dos órgãos alvos e sobrevida eram obtidos, periodicamente. Os participantes foram caracterizados pela sua educação numa tentativa de se estudar como fatores socioeconômicos que poderiam ser influentes nas variáveis estudadas.

\subsubsection{Prevalência de Hipertensão no NHANES II e no HDFP}

Apesar dos achados epidemiológicos de que a pressão arterial é uma variável com distribuição contínua numa população, é necessário categorizar os indivíduos como hipertensos ou normotensos para o propósito do manejo clínico. Existe, ainda, uma implicação de outra graduação quando chamamos os hipertensos de leves, moderados e graves, baseando-nos nos níveis de pressão observados, sem levarmos em 
conta as alterações orgânicas nos órgãos-alvos. A escolha do nível de pressão arterial no qual rotularmos alguém de hipertenso, apesar de arbitrária, tornou-se prática comum quando a pressão arterial de adultos é observada, estando mantida igual ou acima destes pontos de corte. A influência destes pontos de separação na prevalência da hipertensão é ilustrada pelos achados da triagem doméstica do HDFP, na qual cerca de $22 \%$ com diastólicas iguais ou acima de $90 \mathrm{mmHg}$ eram $15 \%$ com $95 \mathrm{mmHg}$ e decresciam, progressivamente, com o aumento do nível de separação entre hipertenso e normotensos até $1,5 \%$, numa diastólica igual ou maior do que $115 \mathrm{mmHg}$. Mais, as estimativas de prevalência foram, marcadamente, reduzidas quando o critério da persistência de elevação de pressão num segundo dia de exames foi introduzido. Trinta e nove por cento de brancos e $30 \%$ de negros, nos quais a triagem em casa foi igual ou maior do que $95 \mathrm{mmHg}$ de diastólica tiveram leituras abaixo de $90 \mathrm{mmHg}$, numa segunda validação na clínica. Esse declínio na prevalência baseado nas leituras em diferentes ocasiões, em oposição a uma única medição, reflete a variabilidade intra-individual da pressão arterial e o fenômeno da regressão à média, quando indivíduos são selecionados para repetidas medidas de pressão baseadas em níveis iniciais elevados. Essas limitações na utilidade das estimativas de prevalência de hipertensão, baseada nas triagens populacionais, feitas numa única ocasião (mesmo se feitas muitas medições em apenas uma ocasião), devem ficar bem claras na mente de quem acompanha a discussão sobre os dados assim obtidos.

A prevalência percentual da diastólica igual ou acima de $95 \mathrm{mmHg}$ por idade, no estudo HDFP e NHANES II, para mulheres estão mostradas na Figura 3. A curva mostrando a ascensão da prevalência com a idade até, aproximadamente, os 50 anos e, após, mostrando um platô e certo declínio é similar (quando se define a hipertensão, categoricamente), àquela observada quando a média de pressões diastólicas é mostrada, como na Figura 1. A prevalência é maior nos pretos do que para brancos em todas as idades, em cada estudo. As diferenças entre as negras e as brancas parecem aumentar com a idade até os 50 anos e, a partir daí, permanecem constantes ou diminuem. A prevalência de diastólica maior ou igual a $95 \mathrm{mmHg}$ foi, aproximadamente, duas a três vezes maior em mulheres negras do que em brancas, em cada um destes estudos. A estimativa de hipertensão foi de algum modo maior no HDFP do que no NHANES, para as amostras de mulheres negras. Os achados foram similares para homens e mulheres quando a prevalência subia até os 50 anos, fazendo um platô e decrescendo, após esta faixa etária (Figura 4). As estimativas de prevalência para os homens brancos foi muito parecida em ambos os estudos. Houve maior variabilidade de prevalência nas diversas faixas etárias entre os homens negros. De qualquer modo, nas idades acima dos 30, os negros tiveram duas a três vezes maior prevalência de elevados níveis de pressão do que os brancos.

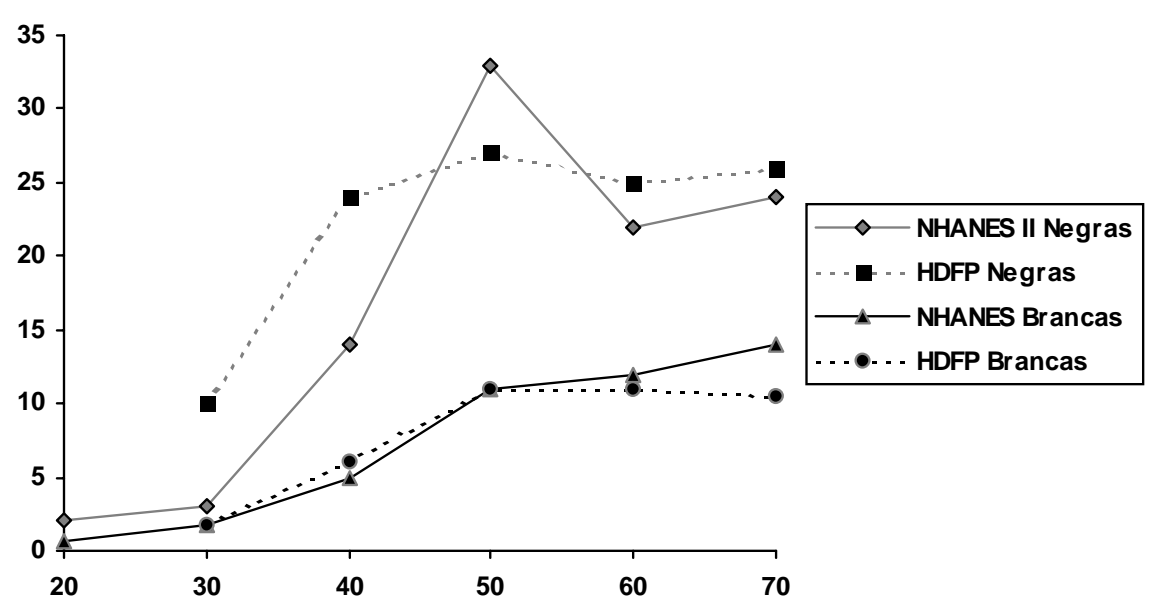

Figura 3. Prevalência percentual de pressão arterial diastólica > ou $=95 \mathrm{mmHg}$, relacionada com a raça, idade e estudo em mulheres, no HDFP e no NHANES II, em mulheres adultas. 
Em adição aos problemas de variabilidade intra-individual e regressão à média, o aumento na detecção, consciência, tratamento e controle da pressão arterial nas populações fazem com que as estimativas de prevalência de hipertensão, baseadas exclusivamente nas medidas de pressão arterial se tornem cada vez menos válidas. Por outro lado, simplesmente, acessar a prevalência pela história de uso de medicação ignora o problema de erro de estimativa da prevalência, induzido pelo tratamento de indivíduos que não necessitariam de tal tratamento. $\mathrm{O}$ fator da motivação pessoal para obter cuidados médicos, associado ao acesso a estes cuidados, custos, variabilidade de qua- lidade e freqüência, podem grosseiramente interferir nas estimativas de prevalência e comparação entre grupos de status social distintos. Como foi visto no NHANES II, entre 1976-1980, aproximadamente $40 \%$ de todas as mulheres com idades entre 55-64 anos nos EUA classificadas como hipertensas, entre 1976-1980, tiveram níveis de diastólica abaixo dos pontos de corte (isto é, estavam controladas). Uma história de consciência da situação foi registrada em $75 \%$ dos hipertensos; $75 \%$ destes, estavam em tratamento e mais de $50 \%$ dos tratados estavam controlados. Não houve diferenças significativas entre brancos e negros quanto à atenção, consciência, tratamento e grau de controle.

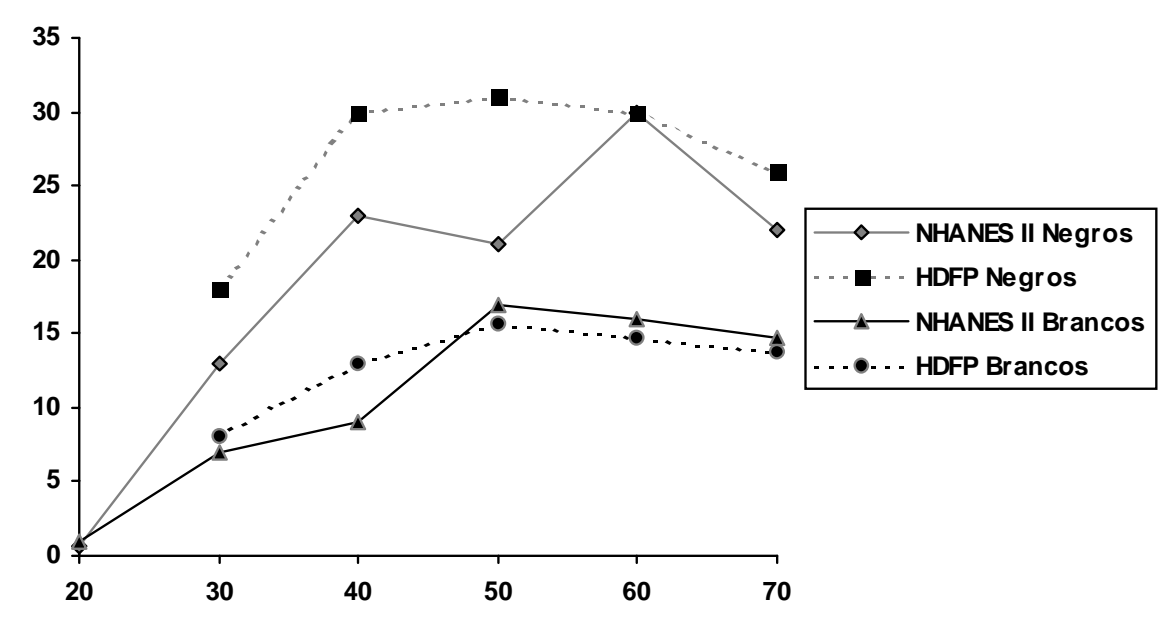

Figura 4. Prevalência percentual de diastólicas $>$ ou $=95 \mathrm{mmHg}$ por idade, raça e estudo, no NHANES II e HDFP, em homens adultos.

\section{RAÇA, EDUCAÇÃO E PREVALÊNCIA DA HI- PERTENSÃO}

Uma associação inversa entre as condições socioeconômicas e os níveis de pressão arterial tem sido relatada em vários estudos epidemiológicos. Estudos de prevalência mostraram associação inversa entre examinandos em estudos baseados em comunidades, clientes de um plano de seguro saúde, servidores civis na Grã Bretanha, funcionários do governo dos EUA, estudantes classificados pela ocupação dos pais, residentes em áreas geográficas caracterizadas pela privação socioeconômica e outros índices de estresse. Uma relação inversa entre o nível educacional e a média de pressão arterial foi, também, apresentada nos dados do NHANES II, no qual as médias de diastólicas foram corrigidas por faixa etária e mostraram variação escalar, conforme as condições educacionais. Comparando-se indivíduos de 13 ou mais anos de escolaridade com aqueles abaixo dos 9 anos, e adultos com idade entre 18-74 anos, a diferença na média de diastólica foi de $2 \mathrm{mmHg}$ para homens brancos, $4 \mathrm{mmHg}$ para mulheres brancas e $6 \mathrm{mmHg}$ para mulheres negras. Não houve evidências de associação do nível educacional com as médias dos homens negros. A diferença na diastólica entre grupos de mesmo status educacional foi sempre menor para homens do que para mulheres. Nas mulheres, as maiores diferenças associadas aos graus educacionais foram observadas para as médias de sistólicas, do que 
para as médias de diastólica: $6 \mathrm{mmHg}$ para mulheres brancas, $12 \mathrm{mmHg}$ para mulheres negras. A diferença de sistólica para diferentes grupos educacionais foi menos pronunciada ou ausente para os homens: homens brancos tiveram uma sistólica média de $3 \mathrm{mmHg}$ superior naqueles com maior escolaridade, comparados àqueles com menos de 9 anos de escola; nos negros a diferença caiu para $1 \mathrm{mmHg}$.

A prevalência de hipertensão definida como diastólica igual ou superior a $95 \mathrm{mmHg}$ e/ou relato de uso corrente de medicação anti-hipertensiva, no estudo HDFP, variou sistematicamente, mensuravelmente e de forma regular com o nível de escolaridade, tanto nos brancos como nos negros. A prevalência de HAS foi maior para os negros do que para os brancos, em cada um dos níveis considerados, e foi maior nos negros que tinham segundo grau, do que nos brancos com menos escolaridade. A relação da prevalência da HAS e escolaridade persistiu entre os triados para o HDFP, após correção por idade, nos brancos e negros; entretanto, a maior diferença relativa de prevalência de HAS, relacionada à escolaridade, foi vista nos grupos mais jovens e mais aparente nos negros, especialmente entre os 30-49 anos de idade. Por exemplo, entre os negros mais jovens neste estudo, aqueles com segundo grau tiveram prevalência de HAS $50 \%$ inferior do que naqueles com menos de 10 anos de educação formal (13,7 \% comparado a 26,6 \%).

A prevalência da HAS na população, em qualquer faixa etária, reflete a incidência de condições antecedentes, sobrevivência nestas condições e eventual tratamento. Dois estudos baseados em comunidades, incluindo todas as raças - O Evans County e o Charleston Heart Study demonstraram uma maior incidência de elevação de pressão arterial nos negros comparados aos brancos; um deles, o Charleston Study, relatou uma maior incidência entre os negros de classe social mais baixa comparados aos negros de classe mais alta. Numerosos estudos têm relatado uma associação positiva e forte entre aumento de pressão arterial e mortalidade, por todas as causas, tanto para os brancos como para os negros. Mas, há maior mortalidade nos indivíduos de classe social baixa nos negros e nos brancos. Isso poderia proporcionar numa maior remoção de hipertensos das camadas sociais mais baixas da população. Assim, associações entre a prevalência de HAS e condições socioeconômicas, detectadas em estudos de coorte são provavelmente estimativas subvalorizadas da força dos antecedentes que levaram às associações. Poucos estudos comunitários multirraciais tentaram identificar e acessar a importância relativa de muitos processos que poderiam explicar a relação entre o status socioeconômico e a HAS. A explicação mais aceitável, derivada dos estudos epidemiológicos sobre os determinantes da HAS, é a da dieta (ex. excesso de sal e calorias na dieta), obesidade, estresse psicológico, exposição a alguns elementos metálicos (chumbo) e uso/abuso do álcool, cada qual sendo mais prevalente nas classes sociais mais baixas. Análises de prevalência da HAS com o status educacional, dentro do estudo HDFP, com correção para índices de massa corporal diminuíram ainda mais o gradiente de pressão arterial e prevalência de HAS, entre os indivíduos brancos; entretanto, o gradiente persistiu entre os negros, particularmente entre os mais jovens. O controle de variáveis como idade, sexo, formação educacional e massa corporal não eliminou a diferença entre brancos e negros, isto é, os negros tiveram uma prevalência sempre maior de HAS, em cada nível destes atributos, comparados aos brancos.

Assim, em resumo, nos EUA, populações existentes lá, aparentemente, apresentam uma correlação inversa entre o nível socioeconômico e a prevalência de HAS tanto para os brancos como para os negros, o que não é nem completamente e nem adequadamente explicado por limitados estudos (particularmente entre os negros) dos níveis correlatos na população. O status socioeconômico provoca um gradiente que é um forte indicador de separação de grupos por prevalência de HAS, tão forte ou mais forte nos negros do que nos brancos. Um efeito residual por ser negro persiste nos estudos epidemiológicos, após o controle de certas variáveis, que poderiam se correlacionar com a pressão arterial.

A estimativa quantitativa do aumento da prevalência da HAS, associada à diminuição do status socioeconômico é dependente do critério usado para categorizar a pressão arterial como elevada. Dada a maior média com o menor status socioeconômico, quanto maior o ponto de separação escolhido, tanto maior o efeito medido da associação dos fatores. Por exemplo, para indivíduos na triagem do estudo HDFP com diastólica entre 90-104 (ou seja, aqueles com menos severa forma de HAS, também chamada de leve), os índices de prevalência foi de 1.1 a 1.3 , em contraste com os mais baixos - os verificados nos indivíduos com mais elevado grau de escolaridade. Em 
contraste, os índices de prevalência entre os grupos estratificados por sexo e raça foram maiores para a categoria de diastólicas, maiores ou igual a $115 \mathrm{mmHg}$ (chamada de HAS severa): para homens brancos, 1.3; para mulheres brancas, 1.7; para homens negros 2.1; e para mulheres negras 2.0. Não só foi mais freqüente a HAS nos com status socioeconômico baixo, mas a distribuição dos níveis de pressão arterial esta deslocada em direção aos níveis mais elevados, resultando em maior prevalência e maior severidade da HAS, com as seqüelas associadas acometendo órgãos-alvos, com consequiente maior mortalidade e morbidade.

\section{A EPIDEMIOLOGIA CLÍNICA DA HIPERTEN- SÃO, EM RELAÇÃO AO STATUS SOCIOECO- NÔMICO E RAÇA}

A experiência dos pacientes destinados ao grupo refered-care do estudo HDFP nos dá uma oportunidade para descrever o curso clínico da HAS, detectada entre os indivíduos de diferentes status socioeconômico e raça oriundos de triagens em 14 populações nos EUA, durante o período de 1973-1974, e em 5 anos de seguimento desde 1975 até 1979. Após obedecer o critério da pressão arterial, ou seja, diastólica igual ou superior a $95 \mathrm{mmHg}$ nas medições feitas nas casas, e igual ou superior a $90 \mathrm{mmHg}$, quando repetidas as medições em clínicas, estes hipertensos eram agrupados usando o critério de elegibilidade adicional à capacidade de comparecer às visitas na clínica. Foram, então, randomizados nos grupos de tratamento escalonado e de tratamento regular. Os pacientes do grupo referidos ao tratamento regular tiveram sua pressão arterial avaliada e tratada pela sua fonte usual de cuidado médico. A experiência com este grupo será descrita em termos de alcance do alvo de pressão arterial durante os 5 anos de seguimento, à reversão da hipertrofia do ventrículo esquerdo (HVE) naqueles que tinham esta condição na triagem ao estudo, a incidência de HVE, durante os 5 anos de seguimento, naqueles pacientes que não tinham esta condição na entrada ao estudo e mortalidade nos indivíduos brancos e negros correlacionados com escolaridade.

\subsection{Meta tensional nos pacientes do grupo de tra- tamento regular}

O desenho do estudo HDFP proporcionou uma meta precisa para a pressão arterial, baseada na pres- são de entrada em cada participante. O HDFP definiu pressão arterial, a ser alcançada como $90 \mathrm{mmHg}$ de diastólica para aqueles que ingressavam no estudo, com níveis superiores a $100 \mathrm{mmHg}$ e um decréscimo de no mínimo $10 \mathrm{mmHg}$ para aqueles que entravam com diastólica entre 90 e $99 \mathrm{mmHg}$. A proporção de indivíduos sob tratamento, em serviços na comunidade e que alcançaram tais metas cresceu dentro de cada grupo considerado, em todas as raças e sexos durante os 5 anos de seguimento. A percentagem de hipertensos com a pressão arterial na meta estabelecida, quando do final do estudo, foi de 38 para homens pretos; 41, para homens brancos; 44, para mulheres negras e 52, para mulheres brancas. Em outras palavras, a pressão arterial foi mais baixa e a percentagem de alcance do gol terapêutico foi maior para as mulheres do que para os homens e para os brancos comparados aos negros, nas condições de tratamento regular. Mulheres brancas tiveram os maiores índices de alcance da meta de pressão arterial. Estas percentagens de hipertensos, controlados pelo tratamento usual não foram diferentes daquelas observadas pelos dados obtidos no estudo NHANES II.

\subsection{Prevalência de HVE entre os hipertensos com menor severidade em relação à escolaridade}

Após a elegibilidade através da triagem doméstica, os candidatos à randomização para o estudo do HDFP, os indivíduos foram avaliados, clinicamente. Incluiu-se um ECG para avaliar a possível presença de HVE, pelo critério do Código de Minnesota, o qual foi um dos testes feitos para detectar evidência de danos em órgãos-alvos relacionados à HAS. A prevalência percentual de HVE em homens hipertensos (diastólica entre 90-104 mmHg), relacionada à educação escolar mostrou que, entre os homens, no ingresso ao estudo, a prevalência de HVE aumentou duas vezes com o decrescer do nível de escolaridade e ficou perto de duas vezes elevada, novamente, nos negros com menos escolaridade. Assim, no grupo dos indivíduos com idades entre 30-69 anos e menos HAS, também referidos como leves, a prevalência da HVE não foi significativa e variou, consistentemente, com o status socioeconômico. A HVE, como indicador de dano em órgão-alvo, foi ela mesma um fator de risco independente da HAS para mortalidade e morbidade, e é tida como um reflexo do efeito da combinação integrada e cumulativa dos níveis prévios de pressão arterial. 
O ajuste de idade para a estimativa de prevalência de HVE, na entrada do estudo, variou com relação à escolaridade nos brancos $(2.1 \%$ em homens com escolaridade mais elevada; $2.6 \%$ para aqueles com segundo grau e $3.4 \%$ para homens brancos com escolaridade mais baixa) e foi mais do que duas vezes prevalente $(8.2 \%)$ em homens negros, com baixa escolaridade. A média de pressão arterial entre os indivíduos que ficaram entre $90-104 \mathrm{mmHg}$, no ingresso ao estudo, não variou entre brancos em termos de distribuição por escolaridade mas foi de $1 \mathrm{mmHg}$ maior na diastólica média no grupo de homens negros, comparados aos brancos no estrato com níveis de escolaridade mais elevado. Assim, a associação observada entre a prevalência de HVE e o nível de escolaridade não foi atribuível às diferenças de níveis de pressão arterial nos indivíduos situados na faixa de pressão de 90 - $104 \mathrm{mmHg}$ e, provavelmente, reflete a distribuição diferencial dos determinantes outros, do que somente a pressão arterial para HVE no sub grupo social e/ou o surgimento prévio, a duração mais longa e o resultante efeito cumulativo de pessoas-anos, quanto aos níveis de pressão arterial, nas camadas mais baixas do estrato social.

\subsection{Regressão da HVE nos pacientes do trata- mento regular}

A reavaliação daqueles pacientes sob tratamento usual e que sobreviveram aos 5 anos de seguimento deu a possibilidade de se acessar as evidências de reversão e regressão da HVE. A incidência dentro do grupo, com tratamento usual, foi um bocado alta para mudanças eletrocardiográficas, mostrando regressão da HVE para a normalidade. A magnitude foi de $57 \%$ para homens brancos, $51 \%$ para homens negros, $48 \%$ para mulheres brancas e $38 \%$ para mulheres negras. Entre aqueles com ondas R, pronunciadas no ECG, e sem evidências de anormalidades no segmento ST ou onda $\mathrm{T}$, na triagem ao estudo, a taxa de reversão ao normal em 5 anos de seguimento sob tratamento regular foi de $56 \%$ para homens brancos, $43 \%$ para negros, $47 \%$ para brancas e $40 \%$ para negras. Assim apesar de a proporção de hipertensos, com evidências eletrocardiográficas, consistentes com HVE os quais reverteram a normalidade tenha sido tão alta sob condições de tratamento regular, a ocorrência foi maior nos brancos do que nos negros. Apesar da avaliação por ECG ser menos do que desejável para validar-se a presença de HVE e os testes de reproducibilidade, na ausência de tratamento, não terem sido feitos, mesmo assim a HVE foi de valor preditivo significante para mortalidade; mais ainda, as observações sugeriram melhor diferença entre os negros e brancos, sob as mesmas condições de tratamento.

\subsection{Incidência de HVE nos pacientes do trata- mento usual}

Durante os 5 anos de seguimento dos indivíduos, que foram referidos ao tratamento usual, três medidas da progressão das evidências eletrocardiográficas de HVE foram acessadas entre os com e os sem ondas $\mathrm{R}$ altas e livres dos critérios de HVE, nos traçados de base. A progressão da HVE entre os com ondas R elevadas no traçado basal foi de $7 \%$ nos homens e $11 \%$ nas mulheres e houve diferenças significativas entre brancos e negros. Por outro lado, a emergência de ondas $\mathrm{R}$ elevadas nos 5 anos de seguimento, entre indivíduos livres, tanto das ondas $\mathrm{R}$ elevadas como das evidências de HVE, no traçado basal, foi maior nos homens comparados às mulheres e, marcadamente, maiores nos negros do que nos brancos: os negros tiveram três vezes mais "chances" de desenvolver HVE do que com os brancos e as mulheres negras tiveram 2 vezes e meia a mais do que as brancas. A taxa de surgimento de ondas R, em 5 anos de seguimento de indivíduos com ECG normal, na entrada ao estudo, oscilou de $2 \%$ em mulheres até $10 \%$ em homens negros. $\mathrm{O}$ aparecimento de critérios para HVE no ECG em hipertensos, com ECG basal normal, foi similarmente baixo para homens e mulheres, porém, discretamente mais elevado nos negros (2\%) e nos brancos $(1 \%)$.

\subsection{Mortalidade por todas as causas e status so- cioeconômico nos pacientes sob tratamento usual}

A evidência revisada até aqui indica uma forte associação entre o status socioeconômico e a prevalência de HAS; a severidade da hipertensão foi indicada pelos níveis tensionais, bem como os danos nos órgãos-alvos, tais como HVE. Existem, também, evidências indicando que os índices de cuidados com a HAS variaram, conforme o status socioeconômico e que o comparecimento à clínica, uso de drogas e o alcance da meta tensional, desenhada para o estudo, variaram com os indicadores de status socioeconômico nas condições do tratamento usual. O prognóstico da HAS, sob estas condições seria, portanto, grosseiramente influenciado pela situação socioeconômica. 
Isso, de fato, é o que foi observado, durante o seguimento dos participantes referidos ao grupo para tratamento usual HDFP.

$\mathrm{O}$ risco de morte variou, marcadamente, entre os hipertensos de diferentes camadas socioeconômicas. Entre os participantes do HDFP randomizados e referidos de volta aos seus costumeiros serviços de saúde (tratamento usual), a mortalidade após 5 anos, ajustada por idade e sexo, entre os brancos subiu de $3,8 \%$ para os hipertensos com mais do que o segundo grau completo, para 5,6 \% para os graduados no segundo grau e 7, $1 \%$ para aqueles com menos escolaridade. Para os negros, o aumento foi de 5,5\% para os com segundo grau completo, $6,9 \%$ nos graduados com segundo grau e 11, $7 \%$ nos abaixo disso. Assim, sob condições de tratamento regular usual na comunidade, estudada pelo HDFP, durante o período de 5 anos de seguimento, houve aproximadamente duas vezes mais aumento de risco de morte nos hipertensos, condicionada na situação socioeconômica estabelecida, indiretamente, pela escolaridade. Hipertensos negros experimentaram índices de mortalidade mais elevados do que os brancos, em cada nível de escolaridade considerado. Tanto nos negros como nos brancos, aqueles com menor grau de escolaridade tiveram taxas de mortalidade corrigida por idade e sexo mais elevada na proporção de duas vezes, comparadas àqueles com escolaridade superior. A associação entre mortalidade, em 5 anos, e escolaridade persistiu e foi estatisticamente significativa entre os hipertensos, triados para o grupo de tratamento usual, mesmo após ajuste de multivariáveis para variáveis, controle que existia numa distribuição desigual na entrada ao estudo e que poderia afetar o prognóstico destes indivíduos.

A importância do status socioeconômico para o prognóstico da HAS, sob condições de tratamento regular e usual, pode ser melhor apreciada pela análise de indivíduos com pressão arterial basal, na faixa dos 90 a $104 \mathrm{mmHg}$, isto é, naqueles com HAS de menor severidade, também erroneamente chamados de leves. Restringindo o seguimento nos homens, sem medicação, e livres de HVE na entrada do estudo, verifica-se um gradiente considerável de aumento da mortalidade, variando de $4,4 \%$ para homens brancos com mais do que o segundo grau escolar até $7,4 \%$, e $7,2 \%$ para os brancos com somente o segundo grau ou abaixo disso, respectivamente, e 14,9\% entre negros, com menos do que o segundo grau.

\subsection{Status socioeconômico e epidemiologia clíni- ca nos pacientes do tratamento escalonado}

A randomização dos hipertensos, assegurada pela triagem na comunidade tanto para o tratamento regular (usual) como para o especial (escalonado), no HDFP, proveu a oportunidade para acessar o efeito do tratamento escalonado na redução ou eliminação da influência dos fatores socioeconômicos sobre a morbidade e mortalidade, observada em outros grupos de hipertensos. Existem restrições quanto a testar a eficácia do tratamento escalonado, separadamente, dentro de cada faixa socioeconômica. Esse tipo de análise envolvendo subgrupos foi feita à parte do desenho original do estudo e resultou que alguns subgrupos tiveram um tamanho amostral reduzido, sua escolha foi feita de forma um tanto arbitrária e as comparações múltiplas reduzem a validade de tal análise. A eficácia geral do programa escalonado foi demonstrada mais rigorosamente, preservando-se a randomização original do desenho do estudo. Adicionalmente, entretanto, houve uma oportunidade de se descrever a relação entre a mortalidade e o status socioeconômico, sob condições de tratamento escalonado como um estudo observacional da epidemiologia clínica.

A proporção de participantes alcançando a meta terapêutica foi consistentemente maior no tratamento escalonado comparado ao usual, tanto para raças, sexo e faixas etárias distintas, em cada ano do seguimento. As diferenças de respostas raciais foram observadas mais entre os homens no estudo escalonado comparado ao regular. Ao contrário, no escalonado, a diferença entre brancos e negros nas mulheres foi consistentemente menor a cada ano de seguimento (4-6\%). Controle de multivariáveis e de modelo indicaram que o status educacional contribuiu para a adesão às visitas na clínica, adesão ao tratamento e alcance da meta terapêutica. Apesar de todo o aumento de uso de medicamentos que o programa escalonado induziu, tanto quanto a redução da pressão arterial conseguida, um efeito residual da influência do status socioeconômico persistiu.

\subsection{Mortalidade e status socioeconômico no gru- po com tratamento escalonado}

Em contraste ao gradiente invertido, marcado da mortalidade em associação ao nível educacional observado no grupo de tratamento usual, no com tratamento escalonado ocorreu, somente, uns $18 \%$ mais 
de mortalidade nos educados até o nível do segundo grau, comparados com os de maior nível educacional, e houve uma mortalidade muito similar entre aqueles com escolaridade superior ao segundo grau, comparados aos graduandos neste nível. As diferenças não foram estatisticamente significantes. A mortalidade de todas as causas, observada ao longo dos 5 anos de seguimento, no grupo escalonado, foi maior do que aquela observada no grupo de tratamento regular entre os participantes com educação, tanto em graduandos do segundo grau, como acima disto. A estimativa destas diferenças, entretanto, considerando a formação amostral, foi pequena em magnitude, com intervalos de confiança bastante amplos ao redor delas e incluíram o caso da nulidade da não diferença. Em contraste, a diferença nos índices de mortalidade, entre os grupos escalonado e regular, $2 \%$ mais baixa no escalonado corrigindo para raça, sexo e idade, entre os com escolaridade mais baixa do que o segundo grau, foi estatisticamente significante. Assim, a análise da mortalidade indica que o programa escalonado eliminou o gradiente socioeconômico na mortalidade, e não só foi efetivo em reduzir a mortalidade geral no estudo, mas também sugeriu que sua eficácia foi máxima naqueles indivíduos com a mais baixa escolaridade. Adicionalmente, indicou que o efeito do tratamento escalonado, comparado ao regular, não foi significativamente diferente entre os vários níveis educacionais envolvidos no estudo. Mais, aproximadamente $44 \%$ do excesso da mortalidade, no grupo regular comparado ao escalonado, foi estatisticamente explicada pelas diferenças na pressão arterial, registrada em ambos os grupos com controle mais eficaz no escalonado.

A eliminação do gradiente de mortalidade, por efeito do status socioeconômico, no grupo escalonado foi observado tanto para os brancos como para os negros. A eficácia do tratamento escalonado foi demonstrada tanto em brancos como em negros e foi, sugestivamente, maior nos negros do que nos brancos. Entretanto, o grupo escalonado do estudo HDFP não eliminou o excesso de mortalidade nos negros. Desigualdades no risco de doenças adicionais associadas à HAS parecem ter persistido e desempenhado um papel notável.

\subsection{Distribuição geográfica da mortalidade re- lacionada à hipertensão e à escolaridade}

Estudos epidemiológicos observacionais, experimentais e ensaios clínicos indicam que a HAS em associação ao status socioeconômico é o maior determinante de mortalidade nas populações consideradas, com enorme potencial de reversão destes índices. As estimativas deste potencial foram derivadas de uma série de estudos epidemiológicos, baseados em comunidades específicas. O risco populacional da HAS associado à mortalidade é uma estimativa de potencial de redução na mortalidade na população, em geral, se as taxas nos hipertensos forem reduzidas para níveis similares daqueles não hipertensos. É o produto e reflete a contribuição de ambos, prevalência de hipertensos numa população e o risco excessivo entre os próprios hipertensos comparados aos não hipertensos. Dados estes componentes é óbvio que o risco populacional será maior nos com mais baixa classificação na pirâmide social, comparados aos mais elevados socialmente, maior nos brancos do que nos negros. Essa conclusão pode ser retirada dos dados de estudos como o de Evans County, biracial e baseado numa comunidade específica. Dividindo a população quanto ao risco de mortalidade atribuível à HAS pela taxa geral de mortalidade, quantifica-se a fração da mortalidade por todas as causas, associada à HAS, nas populações e provê uma estimativa da redução percentual na mortalidade, por todas as causas, observável se a taxa de mortalidade nos hipertensos for reduzida para níveis similares aos dos não hipertensos. Essa fração foi considerável no estudo de seguimento de Evans County e foi maior nos negros do que nos brancos, particularmente maior nas mulheres negras. As estimativas baseadas numa mortalidade de 10 anos de seguimento foi de, aproximadamente, $25 \%$ para homens e mulheres, tanto em brancos como em negros e de $50 \%$ para mulheres negras. Essas frações excessivamente altas de mortalidade, por todas as causas, associada à HAS não é necessariamente generalizável para todas as populações, por causa da alta prevalência de HAS nesta comunidade em particular, além do alto risco cardiovascular, experimentado em populações do sul dos EUA, no período de 1960-1970. De qualquer modo, estudos epidemiológicos, indicam uma considerável contribuição da HAS na mortalidade geral na população e a contribuição diferente em grupos, socialmente, diferentes com um considerável potencial de reversão. A prevalência da HAS e sua importância prognóstica associada contribuíram para a notável fração de mortalidade por todas as causas, uma fração ainda maior nos negros do que nos brancos e, particularmente, maior nas negras. 
Uma série de especulações como extensão destes achados, tais como explicações de variações de tempo e espaço na incidência de doenças cardiovasculares e sua mortalidade foram sugeridas $\mathrm{O}$ forte risco que oferece a própria HAS para acidentes vasculares encefálicos (AVE) foi bem documentado. Uma revisão de vários estudos clínicos indica que o tratamento anti-hipertensivo resultando numa redução média de $5 \mathrm{mmHg}$ de diastólica pode reduzir a mortalidade por AVEs, na ordem de 40-45 \%. Ao passo que, menos consistentemente do que a redução na mortalidade por AVEs, os achados clínicos indicam que existe, também, uma redução na mortalidade em geral, por todas as causas, após tratamento anti-hipertensivo efetivo. A mortalidade por AVEs tem declinado nos EUA e em outras partes do mundo, muito antes da disponibilidade e do largo uso das medicações anti-hipertensivas. Entretanto, uma aceleração considerável no declínio observado tem sido notável, particularmente após 1970, coincidentemente após a introdução de programas educacionais para a saúde tais como o U.S. Hypertension Education Program (1972) e sua abrangência crescente na população, provocando maior alerta sobre a HAS, seu controle, tratamento e seguimento. O declínio da mortalidade tem sido maior para mulheres negras, consistentemente às previsões derivadas das estimativas de risco populacional.

Variações geográficas na distribuição das mortes atribuíveis a AVEs, doenças cardiovasculares, em geral, e por todas as causas, têm sido notadas nos EUA e Europa, nas últimas décadas. Tem havido um declínio marcado na mortalidade cardiovascular, particularmente a por AVEs, nos últimos 20 anos, com alguma variação na distribuição das mortes por região e por tempo considerado. Entretanto, alguma similaridade nos padrões geográficos tem persistido ao longo do tempo, com maiores índices de mortalidade por causas cardiovasculares na região sudeste dos EUA, por exemplo. Existe, também, alguma similaridade espacial na distribuição de mortalidade por AVEs e de mortalidade por todas as causas. Essa distribuição espacial é similar àquela dos padrões de status socioeconômico em regiões socioeconômicas mais problemáticas, tanto nos EUA como em outras partes do globo. As áreas socioeconômicamente diferentes são variadas dentro de um mesmo país. A distribuição do grau de educação dos residentes de determinadas áreas coincide com a distribuição de mortalidade por doenças cardiovasculares e por todas as causas. Por exemplo, entre homens entre 45-65 anos no tempo do censo americano de 1980, a similaridade de padrões de distribuição resultou numa correlação ecológica entre a mortalidade para AVEs, por doenças cardiovasculares, em geral, por mortalidade de todas as causas e a característica socioeconômica do lugar de residência com a magnitude variando desde 0,6 até 0,7 . Estes achados estendidos à comunidade, podem mostrar a importância do status socioeconômico relacionada com o HAS e com a mortalidade. Apesar de que associações ecológicas não podem ser diretamente atribuídas às variáveis em questão, já que as medidas estão sendo comparadas entre áreas de condições econômicas que são propriedades da população das regiões derivadas de médias de grupos mais do que do risco de morte de cada indivíduo, em relação ao seu status socioeconômico, os achados são consistentes com os dados observados em diversos estudos clínicos e epidemiológicos.

Em resumo, a relação inversa existente entre o status socioeconômico e a mortalidade por todas as causas parece ter sido eliminada em hipertensos negros, entre 30-69 anos, na totalidade dos componentes combinados do grupo escalonado do estudo HDFP. As análises estatísticas sugerem que uma larga fração desta redução na mortalidade é atribuível à redução dos níveis de pressão arterial "per se"; entretanto, outros elementos no programa podem também ter contribuído. A existência de uma associação entre o status socioeconômico e a variação geográfica na mortalidade por AVEs, bem como a prevalência de HAS, severidade da elevação dos níveis de pressão arterial, prevalência de danos em órgãos-alvos da HAS, participação e adesão aos programas de cuidados de saúde, uso de remédios, diminuição da pressão arterial e mudanças comportamentais, sociais e biomédicas, todos eles contribuem para que uma pervasiva influência do status socioeconômico, medido pela escolaridade, na epidemiologia clínica da HAS na população, seja como foi documentada nestes vários estudos. A capacidade de um programa de intervenção mais amplo, com reduzidas barreiras de acesso ao tratamento anti-hipertensivo para impactar e modificar algumas destas várias variáveis, resultou na aparente eliminação do gradiente de mortalidade por distinções sociais entre hipertensos negros e brancos tratados, com consequiente redução da mortalidade, em geral. Isso apresenta um modelo que oferece um desafio para uma aplicação num nível populacional para eliminar iniquiidades sociais com o objetivo de melhor controle da HAS e suas consequiências, em outras palavras, a prevenção primária da HAS passa pela melhoria das condições de vida e vice-versa. 
SPRITZER N. Epidemiology of hipertension. Medicina, Ribeirão Preto, 29: 199-213, apr./sept. 1996.

ABSTRACT: Prevalence of hypertension, mainly that reported on community based studies is evaluated with special concern on the differences of rates among diferent groups of hypertensives: blacks, withs, males, females, more and/or less educated subjects, young, aged. Consequences for primary prevention are discussed.

UNITERMS: Hypertension. Cardiovascular Diseasis

\section{LITERATURA RECOMENDADA}

1 - HYPERTENSION DETECTION AND FOLLOW-UP PROGRAM COOPERATIVE GROUP. Blood Pressure studies in 14 communities: a two-stage screen for hypertension. JAMA 237; 2385-2391, 1977.

2 - DRIZD T; DANNENBERG AL \& ENGEL A. Blood pressure levels in persons 18-74 years of age in 1976-80, and trends in blood pressure from 1960 to 1980 in the United States. U.S. Government Printing Office, Washington, 1986 (NCHS Series 11 \# 234).

3 - HYPERTENSION DETECTION AND FOLLOW-UP PROGRAM COOPERATIVE GROUP. The Hypertension Detection and Follow-up Program. Prev Med 5: 207-215, 1976.

4 - HYPERTENSION DETECTION AND FOLLOW-UP PROGRAM COOPERATIVE GROUP. Five-year findings of the Hypertension Detection and Follow-up Program: I. Reduction in mortality of persons with high blood pressure, including mild hypertension. JAMA 242: 2562-2571, 1979.

5 - HYPERTENSION DETECTION AND FOLLOW-UP PROGRAM COOPERATIVE GROUP. Five-year findings of the Hypertension Detection and Follow-up Program: II. Mortality by racesex and age. JAMA 242: 2572-2577, 1979.
6 - HYPERTENSION DETECTION AND FOLLOW-UP PROGRAM COOPERATIVE GROUP. Five-year findings of the Hypertension Detection and Follow-up Program: III. Reduction in stroke incidence among persons with high blood pressure. JAMA 247: 633-638, 1982.

7 - HYPERTENSION DETECTION AND FOLLOW-UP PROGRAM COOPERATIVE GROUP. Race, education and prevalence of hypertension. Am J Epidemiol 106: 351-361, 1977.

8 - HYPERTENSION DETECTION AND FOLLOW-UP PROGRAM COOPERATIVE GROUP. Prevention and reversal of left ventricular hypertrophy with antihypertensive drug therapy. Hypertension 7: 105-107, 1985.

9 - HYPERTENSION DETECTION AND FOLLOW-UP PROGRAM COOPERATIVE GROUP. Educational level and 5year all cause mortality in the Hypertension Detection and Follow-up Program. Hypertension 9: 641-646, 1987.

10 - MACMAHON SW et al. The effects of drug treatment for hypertension on morbidity and mortality from cardiovascular disease: a review of randomized controlled trials. Prog Cardiovasc Dis 29 (supl 1); 99-118, 1986.

Recebido para publicação em 16/01/96

Aprovado para publicação em 25/04/96 